Original

\title{
El indice de masa corporal y la dieta afectan el desarrollo del cáncer de próstata
}

\author{
Constanza M. López Fontana*, Gabriela M. Recalde Rincón*, Diego Messina Lombino*, \\ Ana L. Uvilla Recupero*, Rafael F. Pérez Elizalde*, José D. López Laur,** \\ *Laboratorio de Enfermedades Metabólicas y Cáncer. Facultad de Farmacia y Bioquímica. Universidad Juan \\ Agustín Maza. Guaymallén. Mendoza, Argentina. **Cátedra Clínica Urológica. Facultad de Ciencias Médicas. \\ Universidad Nacional de Cuyo. Mendoza, Argentina.
}

\section{Resumen}

Introducción: El adenocarcinoma de próstata (CaP) es una de las causas más importantes de morbimortalidad en el mundo. La obesidad y los hábitos alimentarios inadecuados favorecerían el desarrollo del CaP.

Objetivo: Analizar y comparar el índice de masa corporal (IMC) y la historia alimentaria, especialmente el consumo de grasas y antioxidantes, entre sujetos con CaP diagnosticado y sujetos libres de enfermedad.

Material y Métodos: fueron seleccionados 40 hombres entre 50 y 80 años: 20 con cáncer de próstata diagnosticado y 20 libres de enfermedad como grupo control, a los cuales se les realizó tacto rectal, medición de antígeno prostático específico, ecografía transrectal y biopsia ecodirigida de próstata; y una entrevista nutricional que incluyó una historia dietética detallada y mediciones antropométricas. El análisis estadístico se realizó mediante Test de Student para muestras independientes $(\mathrm{p}<0,05)$.

Resultados: El IMC en los sujetos con CaP fue superior que en los controles $\left(29,8 \mathrm{~kg} / \mathrm{m}^{2} \mathrm{vs} .27,96 \mathrm{~kg} / \mathrm{m}^{2} ; \mathrm{p}=0,13\right)$ aunque estadísticamente no significativo. Sin embargo, se observó una correlación directa entre el IMC y la agresividad del tumor ( $\mathrm{r}=0,79$; $\mathrm{p}<0.001)$. El consumo de grasas totales, saturadas, monoinsaturadas y poliinsaturadas fue significativamente mayor en los sujetos con $\mathrm{CaP}(\mathrm{p}=0,001)$; mientras que la ingesta de ácidos grasos $\omega 3$, vitamina $\mathrm{C}$ y licopeno fue significativamente menor independientemente del Score de Gleason que presentasen ( $\mathrm{p}<0,05)$.

Conclusiones: Un peso saludable y una alimentación baja en grasas totales, saturadas, monoinsaturadas y poliinsaturadas y rica en ácidos grasos $\omega 3$, vitamina $\mathrm{C}$ y licopeno se asocia a un menor riesgo de CaP.

Palabras clave: Cáncer de próstata. Sobrepeso. Índice de masa corporal. Grasas saturadas. Grasas monoinsaturadas. Grasas poliinsaturadas. Vitamina C. Licopeno.

\section{Body mass index and diet affect prostate cancer development}

\section{Abstract}

Introduction: Prostate cancer (CaP) is one of the most important causes of morbidity and mortality in the world. There is evidence that obesity and inadequate eating habits may promote CaP development.

Objective: To analyze and compare the body mass index (BMI) and the food intake, especially fats and antioxidants, among subjects with $\mathrm{CaP}$ and those free of disease as a control group.

Material and Methods: A sample of 40 men between 50 and 80 years old were selected for the study: 20 with CaP and 20 healthy men as control group. All volunteers underwent a digital rectal examination, prostate specific antigen level, ultrasound and transrectal prostate biopsy, and a nutritional interview where a dietary history and different anthropometric measurements were made. Statistical analysis was performed using the Student $\mathrm{T}$ test for independent samples $(\mathrm{p}<0.05)$.

Results: BMI in the subjects with CaP was higher than in controls $\left(29.8 \mathrm{~kg} / \mathrm{m} 2 \mathrm{vs} .27 .96 \mathrm{~kg} / \mathrm{m}^{2}\right.$, p = 0.13$) \mathrm{but}$ not statistically significant. However, there was a direct correlation between BMI and tumor aggressiveness $(r=0.79$, P <0.001). Total, saturated, monounsaturated and polyunsaturated fat intake was significantly higher in subjects with CaP; while $\omega 3$ fatty acids, vitamin $\mathrm{C}$ and lycopene intake was significantly lower than in controls ( $\mathrm{p}<0.05)$.

Conclusions: A healthy weight and a diet low in total fat, saturated, monounsaturated and polyunsaturated fat and rich in $\omega 3$ fatty acids, vitamin $\mathrm{C}$ and lycopene is associated with a lower risk of CaP.

Keywords: Prostate cancer. Overweight. Body mass index. Saturated fats. Monounsaturated fats. Polyunsaturated fats. Vitamin C. Lycopene.

Subsidio de investigación: Universidad Juan Agustín Maza, Universidad Nacional de Cuyo y Fundación Allende. Las instituciones sólo proporcionaron ayuda financiera sin tener influencia en el estudio. 
$\mathrm{E}$ 1 cáncer de próstata $(\mathrm{CaP})$ es la segunda causa de muerte por enfermedades neoplásicas en los hombres mayores de 50 años en los Estados Unidos. Este tumor suele ser una enfermedad de la edad avanzada y su incidencia aumenta en forma progresiva con los años de vida ${ }^{1,2}$.

Numerosos estudios señalan la existencia de factores exógenos y endógenos, vinculados entre sí, que participan sobre la inducción y progresión tumoral prostática. Entre los primeros, se destacan los factores genéticos (tales como raza negra y antecedentes de CaP en familiares de primer grado) y los hormonales, específicamente los andrógenos ${ }^{3}$. Sin embargo, entre el 80 y el 90\% de los tumores malignos se relacionan con factores exógenos, y un 35\%, con hábitos alimentarios y dietéticos.

Existe evidencia de que los hábitos alimentarios occidentales, tales como el consumo excesivo de alimentos hipercalóricos, ricos en grasas saturadas y pobres en vitamina $\mathrm{E}$, selenio, fibra, licopeno, etc., podrían iniciar, promover o favorecer la progresión del $\mathrm{CaP}^{4-6}$.

Estudios epidemiológicos revelan que la incidencia de CaP latente es similar en hombres de países orientales y occidentales, mientras la incidencia de enfermedad maligna es mayor en hombres norteamericanos de raza negra que en japoneses y chinos ${ }^{7,8}$. Otros investigadores han observado que hay un incremento de la incidencia de CaP en orientales que emigran a los Estados Unidos $^{9}$. Esto sugiere que factores ambientales y nutricionales, tienen un rol en la estimulación del crecimiento del cáncer latente microfocal al cáncer clínicamente detectable ${ }^{5}$.

Por ello, el objetivo del presente trabajo fue analizar y comparar el índice de masa corporal (IMC) y la historia alimentaria, especialmente el consumo de grasas y antioxidantes, entre los pacientes con CaP diagnosticado y sujetos libres de enfermedad como grupo control.

\section{MATERIAL Y MÉTODO}

\section{Población}

La población estuvo constituida por 40 individuos, entre 50 y 80 años de edad, seleccionados al azar a partir de una consulta urológica de rutina teniendo en cuenta los criterios de inclusión y exclusión mencionados en la Tabla 1.

Los voluntarios fueron clasificados en dos grupos:
- Grupo Control: formado por 20 sujetos normales sin riesgo aparente de CaP y sin enfermedad clínicamente ni bioquímicamente detectable. Individuos entre 50 y 80 años de edad, sin síntomas de prostatismo, tacto rectal normal, PSA menor a $4 \mathrm{ng} / \mathrm{ml}$ y próstata menor a 30 gramos.

- Grupo con Cáncer: 20 pacientes con CaP hormonosensibles diagnosticados con biopsia prostática ecodirigida.

\section{Diseño del Estudio}

El trabajo consistió en una consulta urológica de rutina, en la cual se realizó tacto rectal, medición de antígeno prostático específico (PSA), medición del volumen prostático por ecografía transrectal y biopsia ecodirigida de próstata; y una entrevista nutricional donde se efectuó una historia dietética detallada (cuestionario de frecuencia de consumo y recordatorio de $24 \mathrm{~h}$ ) y se evaluó la composición corporal mediante antropometría (peso, talla e IMC).

Todos los hombres que participaron del estudio firmaron un consentimiento escrito a un protocolo previamente aprobado por el Comité de Ética de la Universidad Nacional de Cuyo, Mendoza, Argentina.

\section{Consulta Urológica de rutina}

- Tacto Rectal: realizado por el urólogo en la primera consulta.

- Medición de PSA (Total, libre y su relación): se utilizó el método de laboratorios Abbott. El PSA es producido por las células del epitelio glandular del acino prostático. Cuando la célula neoplásica rompe la membrana basal, pasa a circulación general produciéndose una elevación de éste a valores detectables en sangre. Valor de referencia: hasta 4 $\mathrm{ng} / \mathrm{ml}$.

- Ecografía Transrectal y Biopsia de próstata ecodirigida: se realizó vía transrectal con un transductor de 7,5 Mhz, sin preparación intestinal y con antibiótico terapia una hora antes. En aquellos casos de sospecha de tumor, se introdujo una aguja de punción y se obtuvieron biopsias por sextantes con cilindros de $1,5 \mathrm{~cm}$.

\section{Entrevista Nutricional}

Todos los voluntarios fueron sometidos a una evaluación de la composición corporal mediante antropometría incluyendo peso y talla debidamente estandarizados y se utilizó el IMC para evaluar el estado nutricional. 
Tabla 1. Criterios de inclusión y exclusión

\begin{tabular}{|c|c|}
\hline Criterios de inclusión & Criterios de exclusión \\
\hline Sujetos mayores de 50 años & Sujetos mayores de 80 años \\
\hline Sujetos con nódulos prostáticos & Individuos con próstata mayor a $30 \mathrm{~g}$ \\
\hline PSA menor de $4 \mathrm{ng} / \mathrm{ml}$. & Antecedentes familiares de $\mathrm{CaP}$ \\
\hline \multirow[t]{9}{*}{ CaP diagnosticado } & Tacto rectal sospechoso \\
\hline & Individuos con PIN (neoplasia intraepitelial de próstata) \\
\hline & Sujetos con biopsias reiteradas negativas y PSA elevado \\
\hline & Individuos con hiperplasias atípica de próstata \\
\hline & Sujetos con otro tipo de tumores urológicos asociados. \\
\hline & Trastornos hemorragíparos por biopsias. \\
\hline & Individuos con sonda vesical a permanencia. \\
\hline & Individuos con cáncer prostático antiguo \\
\hline & $\begin{array}{l}\text { Sujetos con enfermedades crónicas como insuficiencia renal, hepática o } \\
\text { respiratoria }\end{array}$ \\
\hline
\end{tabular}

- Peso: provee una evaluación a grandes rasgos de toda la composición corporal. Se midió con el mínimo de ropa y se utilizó una balanza marca CAM.

- Talla: es la medición más comúnmente usada para determinar el peso ideal. Se utilizó tallímetro que forma parte de la balanza ya descrita. Se midió a las personas descalzas, paradas con los pies paralelos en el piso, talones juntos, espalda derecha y ambos brazos colgando naturalmente a los lados del cuerpo y cabeza en plano horizontal.

- Índice de Masa Corporal: explica las diferencias en la composición corporal al definir el nivel de adiposidad, con base en la relación entre peso y talla. La puntuación es la siguiente: 20 a $25 \mathrm{~kg} / \mathrm{m}^{2}$ normal; 25 a $29,9 \mathrm{~kg} / \mathrm{m}^{2}$ sobrepeso; 30 a $35 \mathrm{~kg} / \mathrm{m}^{2}$ obesidad grado I, 35 a $40 \mathrm{~kg} / \mathrm{m}^{2}$ obesidad grado II y más de $40 \mathrm{~kg} / \mathrm{m}^{2}$ obesidad grado III.

Además, se llevó a cabo una evaluación de la historia alimentaria, la cual permitió obtener información sobre los hábitos y pautas de alimentación y determinar la cantidad y calidad de los alimentos consumidos. Se evaluó mediante recordatorio de 24 horas y cuestionario de frecuencia de consumo. Ambos cuestionarios permitieron hacer una estimación más precisa de la ingesta del individuo. Los datos obtenidos fueron volcados en una hoja de cálculos de composición química de los alimentos.

\section{Análisis Estadístico}

Promedios y desviaciones estándar fueron calculados de cada una de las variables; y con el fin de establecer las diferencias y correlación entre los grupos, se utilizó Test de Student estableciéndose como significancia estadística un $\mathrm{p}<0,05$.

\section{RESULTADOS}

La población en estudio estuvo constituida por 20 sujetos controles y 20 sujetos con CaP diagnosticado. A su vez, este último grupo se dividió según el score de Gleason como marcador de agresividad del tumor: 5 presentaron score de Gleason $<6$ (bajo); 7 un score de Gleason 7 (medio) y 8 un score de Gleason 8 (alto).

El IMC fue superior en los sujetos con CaP $(29,80$ $\left.\mathrm{kg} / \mathrm{m}^{2}\right)$ comparado con los controles $\left(27,96 \mathrm{~kg} / \mathrm{m}^{2}\right)$, aunque sin llegar a ser una diferencia estadísticamente significativa $(p=0,13)$. Sin embargo, el IMC resultó mayor a medida que aumentaba el score de Gleason en sujetos con CaP: $24,68 \mathrm{~kg} / \mathrm{m}^{2}$ en los individuos con score de Gleason 6; $29,16 \mathrm{~kg} / \mathrm{m}^{2}$ en aquellos con score de Gleason 7 y 33,56 kg/m² en los pacientes con score de Gleason 8. Esto indica que existe una correlación directa muy significativa entre el IMC y la agresividad del tumor $(\mathrm{p}<0,001)$.

En cuanto al consumo de energía, los pacientes con cáncer mostraron una ingesta calórica significativamente mayor que los controles $(2.352 \pm 363$ kcal/día vs. $1.958 \pm 304 \mathrm{kcal} /$ día; $\mathrm{p}<0,005$ ).

La distribución de macronutrientes en los sujetos con CaP fue similar a lo recomendado para la población general. Sin embargo, al comparar ambos grupos, los sujetos con $\mathrm{CaP}$ presentaron una mayor ingesta de grasas totales y, por consiguiente, una menor ingesta de carbohidratos que los sujetos con- 
troles ( $\mathrm{p}<0,005$; Tabla 2). Además, se observa que el consumo de macronutrientes no mostró diferencias estadísticamente significativas según el score de Gleason ( $p>0,05)$, es decir que la agresividad del tumor no se asoció a la distribución de macronutrientes.

La ingesta de ácidos grasos saturados, monoinsaturados y poliinsaturados fue superior en los sujetos con $\mathrm{CaP}(\mathrm{P}<0,001$; Tabla 3$)$. El consumo de ácidos grasos saturados fue significativamente mayor en los individuos con score de Gleason 8 $(\mathrm{p}<0,05)$, mientras que la ingesta de ácidos grasos monoinsaturados y poliinsaturados no mostró diferencias estadísticamente significativas entre los grupos ( $\mathrm{p}>0,05$; Tabla 3).

Contrariamente, el consumo de ácidos grasos $\omega 3$ Eicosapentaenoico (EPA) y Docosahexaenoico (DHA) fue significativamente mayor en el grupo control que en el grupo con $\mathrm{CaP}(0,065 \pm 0,03 \mathrm{~g} /$ día vs. 0,031 \pm

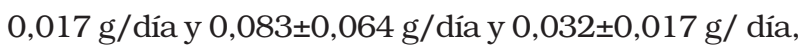
respectivamente) con una diferencia estadísticamente significativa ( $\mathrm{P}=0,002$; Tabla 4). Además, se aprecia que la ingesta de ácidos grasos $\omega-3$ no mostró diferencias estadísticamente significativas según el score de Gleason ( $p>0,05)$.

Finalmente, el consumo de micronutrientes no fue estadísticamente diferente en lo que respecta a la vitamina A, vitamina $\mathrm{D}$, vitamina $\mathrm{E}$ y Selenio (Tabla 5). Sin embargo, la ingesta de vitamina $C$ fue superior en el grupo control comparado con los sujetos con CaP $(144,5 \pm 79,6 \mathrm{mg} /$ día vs. $77,5 \pm 45$ mg/día; $\mathrm{p}<0,05)$; y la ingesta de licopeno también fue mayor en los controles $(3.097 \pm 1415 \mu \mathrm{g} /$ día vs. $860 \pm 730 \mu \mathrm{g} /$ día; $\mathrm{p}<0,005$ )

\section{DISCUSIÓN}

Los resultados obtenidos en este estudio muestran que los individuos con CaP presentaban un IMC levemente mayor con respecto a los controles. Ambos grupos poseían un IMC entre 27,5 y $30 \mathrm{~kg} / \mathrm{m}^{2}$, que según la clasificación de la OMS corresponde a sobrepeso. Al analizar la muestra según el score de Gleason, el IMC fue superior a medida que aumentaba la agresividad del tumor. Estos resultados, al igual que los de otros investigadores, sugieren que existe una correlación directa entre el IMC y la agresividad del tumor ${ }^{10}$. Estudios recientes indican que los hombres obesos presentan tumores más agresivos y avanzados en el momento del diagnóstico, posiblemente debido a que estos pacientes tienen una menor concentración plasmática de PSA con respecto a los hombres normopesos. A su vez, la obesidad interfiere en los métodos de diagnóstico complementarios y en la exploración física, dificul-

Tabla 2. Distribución de macronutrientes

\begin{tabular}{|c|c|c|c|c|c|}
\hline & \multirow[t]{2}{*}{ Control } & \multicolumn{4}{|c|}{ CaP } \\
\hline & & Total & S. Gleason 6 & S. Gleason 7 & S. Gleason 8 \\
\hline Hidratos de Carbono (\%) & $57,1 \pm 4,99$ & $48,65 \pm 6,62$ & $51 \pm 6,18$ & $48,2 \pm 4,52$ & $47,6 \pm 8,61$ \\
\hline Proteínas (\%) & $18,4 \pm 3,2$ & $18,7 \pm 2,37$ & $16,9 \pm 1,62$ & $18,8 \pm 1,38$ & $19,7 \pm 2,95$ \\
\hline Grasas (\%) & $24,5 \pm 3,84$ & $32,68 \pm 5,43$ & $32,1 \pm 4,59$ & $33 \pm 4,45$ & $32,8 \pm 7,12$ \\
\hline
\end{tabular}

Tabla 3. Ingesta de ácidos grasos

\begin{tabular}{|c|c|c|c|c|c|}
\hline \multirow[t]{2}{*}{ Ácidos grasos } & \multirow[t]{2}{*}{ Control } & \multicolumn{4}{|c|}{ CaP } \\
\hline & & Total & S. Gleason 6 & S. Gleason 7 & S. Gleason 8 \\
\hline Saturados (g/día) & $16,25 \pm 4,28$ & $33,71 \pm 9,67$ & $29 \pm 8,87$ & $32,7 \pm 4,86$ & $37,6 \pm 12,45$ \\
\hline Monoinsaturados (g/día) & $22,02 \pm 3,57$ & $28,02 \pm 6,84$ & $27,8 \pm 4,25$ & $26,7 \pm 6,62$ & $29,3 \pm 8,71$ \\
\hline Poliinsaturados (g/día) & $10,04 \pm 3,93$ & $19,5 \pm 7,1$ & $24 \pm 6,88$ & $17,6 \pm 7,95$ & $18,6 \pm 6,06$ \\
\hline
\end{tabular}

Tabla 4. Ingesta de ácidos grasos $\omega-3$

\begin{tabular}{lccccc}
\hline Ácidos grasos $\boldsymbol{\omega}$-3 & Control & Total & S. Gleason 6 & S. Gleason 7 & S. Gleason 8 \\
\hline Ácido Eicosapentaenoico $(\mathrm{g})$ & $0,065 \pm 0,02$ & $0,031 \pm 0,016$ & $0,033 \pm 0,022$ & $0,030 \pm 0,018$ & $0,031 \pm 0,014$ \\
Ácido Docosahexaenoico $(\mathrm{g})$ & $0,083 \pm 0,06$ & $0,032 \pm 0,017$ & $0,033 \pm 0,022$ & $0,030 \pm 0,018$ & $0,034 \pm 0,015$ \\
\hline
\end{tabular}


Tabla 5. Consumo de micronutrientes

\begin{tabular}{lccc}
\hline & $\begin{array}{c}\text { Grupo Control } \\
\text { Media } \pm D E\end{array}$ & $\begin{array}{c}\text { Grupo con CaP } \\
\text { Media } \pm D E\end{array}$ & P \\
\hline Vitamina E (mg/día) & $9,6 \pm 2,7$ & $16,8 \pm 9,5$ & $<0,005$ \\
Vitamina C (mg/día) & $144,5 \pm 79,6$ & $77,5 \pm 45$ & $<0,005$ \\
Selenio (mg/día) & $123 \pm 37$ & $114 \pm 27$ & $>0,005$ \\
Vitamina A (RE) & $1355 \pm 804$ & $1238 \pm 936$ & $>0,005$ \\
Caroteno (RE) & $813 \pm 424$ & $552 \pm 345$ & $>0,005$ \\
Licopeno ( $\mu$ g) & $3097 \pm 1415$ & $860 \pm 730$ & $<0,005$ \\
Vitamina D (UI) & $187 \pm 83$ & $267 \pm 122$ & $>0,005$ \\
\hline
\end{tabular}

importante pues dicha relación está todavía en discusión ${ }^{21}$. Pocos estudios han encontrado que el ácido ascórbico disminuye el riesgo de $\mathrm{CaP}^{22}$. Los resultados de nuestro estudio condicen con las evidencias de que la vitamina $\mathrm{C}$ protege contra el cáncer ${ }^{22}$.

En cuanto al licopeno, los resultados de este estudio concuerdan con los de Grant y Clinton, respecto de que los sujetos con una dieta rica en licopeno tienen un riesgo menor de $\mathrm{CaP}^{23,24}$. Estos estudios anteriores utilizaron, al igual que

tando el diagnóstico y la detección precoz del CaP en estos pacientes $^{11,12}$.

Asimismo, la obesidad está altamente correlacionada con la alimentación, ya que dietas hipercalóricas y ricas en grasas influirían en el desarrollo de $\mathrm{CaP}^{5,13,14}$. Nuestros resultados muestran que la historia dietaria es diferente en los individuos con $\mathrm{CaP}$ comparada con la de los controles. La ingesta calórica fue superior en los sujetos con cancer y la distribución de macronutrientes también varió entre los dos grupos. El consumo de grasas totales, saturadas, monoinsaturadas y poliinsaturadas fue superior en el grupo con $\mathrm{CaP}$ independientemente de la agresividad del tumor. En este estudio nuevamente se confirma la relación positiva entre el consumo de lípidos y el cáncer, resultado que coincide con otros estudios epidemiológicos ${ }^{13-17}$. No obstante, los mecanismos moleculares que subyacen la asociación entre grasa dietaria y tumor siguen aún sin establecerse. Posiblemente estos mecanismos estarían asociados con un alto consumo de colesterol y ácidos grasos específicos (saturados, trans, etc.), el estrés oxidativo, el aumento de andrógenos y a la exposición a pesticidas, ya que la mayoría de ellos son liposolubles y por lo tanto, serían vehículo de sustancias cancerígenas.

Además, y al igual que otros estudios recientes, el presente trabajo muestra la relación existente entre el CaP y el menor consumo de ácidos grasos $\omega-3$ y sugiere una disminución del riesgo de este tumor con el consumo de EPA Y DHA ${ }^{18-20}$.

Los componentes de la dieta que se han asociado con un menor riesgo de $\mathrm{CaP}$ son la vitamina $\mathrm{C} \mathrm{y}$ el licopeno. La vitamina $C$ mostró una asociación negativa con la presencia de cáncer, lo cual es el nuestro, encuestas de frecuencia alimentaria y mostraron que el licopeno tiene un efecto inversamente proporcional con el CaP. El método de preparación del tomate basado en su cocción en aceite, podría aumentar la efectividad del licopeno como protector de esta neoplasia.

El efecto de la vitamina $E$ en la carcinogénesis prostática aún es controvertido. Diversos trabajos sugieren un efecto protector de la vitamina $\mathrm{E}$ por su poder antioxidante ${ }^{13,25}$ mientras que otros investigadores no han encontrado tal beneficio ${ }^{25-27}$. El resultado obtenido en este estudio muestra un aumento en la ingesta promedio de vitamina $\mathrm{E}$ a medida que crece el riesgo de cáncer. Este resultado paradójico puede explicarse por el hecho de que esta vitamina se ingiere acompañada de agentes procancerígenos como las grasas, dado que su fuente natural son los aceites. De manera que su aumento en los grupos de riesgo y con CaP se puede deber a la mayor ingesta de lípidos, lo que se constató al observar que ninguno de los sujetos consumía vitamina E sintética. Si se considera la ingesta de vitamina $\mathrm{E}$ por gramo de grasa consumida, se observa que los dos grupos consumen similar cantidad.

El consumo de Selenio no varió según el grupo de estudio. La ingestión de este micronutriente se ve influenciada por la fuente dietaria ya que depende de la cantidad presente en los suelos de origen del alimento. Esto dificulta la estimación de su consumo, debido a que las tablas de composición de alimentos no contemplan las variaciones territoriales $^{28}$.

Con respecto a la vitamina $\mathrm{A}$, hasta el momento, los resultados de los estudios son conflictivos $^{22,29}$. 
Una explicación posible para estos resultados puede ser la fuente de vitamina A: en Asia y otras áreas con baja incidencia de CAP. Allí esta vitamina es obtenida principalmente de los vegetales, mientras que en occidente la fuente alimentaria es grasa. En este trabajo, no se encontró una relación entre la vitamina A y el CaP probablemente por esta causa, por lo cual se sugieren estudios adicionales en los cuales se especifique la fuente alimentaria de esta vitamina.

En conclusión, el presente estudio permitió relacionar el IMC y la ingesta de macro y micronutrientes con el CaP, y se concluyó que una alimentación saludable se asocia a una menor presencia de este tumor maligno. Así, el valor calórico total y el porcentaje de grasas totales correspondiente son mayores en los sujetos con CaP, mientras que la ingesta de ácidos grasos eicosapentaenoico y docosahexaenoico, vitamina $\mathrm{C}$ y licopeno es menor en este grupo respecto a los controles.

\section{REFERENCIAS}

1. Freedland SJ, Aronson WJ. Obesity and prostate cancer. Urology. 2005;65(3):433-439

2. O־Malley RL, Teneja SS. Obesity and prostate cancer. Can J Urol. 2006; 13(2):11-17.

3. Chan JM, Gann PH, Giovannucci EL. Role of diet in prostate cancer development and progression. J Clin Oncol. 2005;23(32):8152-8160.

4. Wirth MP, Hakenberg OW. Prevention of prostate cancer. Dtsch Med Wochenschr. 2005;130(36):2002-2004.

5. Romero Cagigal I, Ferreruelo Alonso A, Berenguer Sanchez A. Dieta y cáncer de próstata. Actas Urol Esp 2003; 27(6): 399-409.

6. Stamatiou K, Alevizos A, Mariolis A, Sofras F. Dieta mediterránea y cáncer de próstata. Actas Urol Esp 2006; 30(3):340.

7. Williams H, Powell IJ.Epidemiology, pathology, and genetics of prostate cancer among African Americans compared with other ethnicities. Methods Mol Biol. 2009; 472:439-453.

8. Baade PD, Youlden DR, Krnjacki LJ. International epidemiology of prostate cancer: Geographical distribution and secular trends. Mol Nutr Food Res. 200953(2):171-184.

9. Shimizu H, Ross RK, Bernstein L, Yatani R, Henderson BE, Mack TM Cancers of the prostate and breast among Japanese and white immigrants in Los Angeles County. Br J Cancer. 1991;63(6):963-966.

10. Demark-Wahnefried W, Moyad MA. Dietary intervention in the management of prostate cancer. Curr Opin Urol. 2007;17(3):168-174.

11. Banez LL, Hamilton RJ, Partin AW, Vollmer RT, Sun L, Rodríguez C, et al. Obesity-related plasma hemodilution and PSA concentration among men with prostate cancer. JAMA. 2007; 298(19):2275-2280.

12. Pruthi RS, Swords K, Schultz H, Carson CC 3rd, Wallen EM. The impact of obesity on the diagnosis of prostate cancer using a modern estended bipsy scheme. 2009 Feb;181(2):574-577.

13. Giovannucci E, Liu Y, Platz EA, Stamfer MJ, Willett WC. Risk factors for prostate cancer incidence and progression in the health professional follow-up study. Int J Cancer. 2007; 121(7):1571-1578.
14. Stamatiou K, Delakas D, Sofras F. Mediterranean diet, monounsaturated: saturated fat ratio and low prostate cancer risk. A myth or a reality? Minerva Urol Nefrol. 2007;59(1):59-66.

15. Thompson IM. Chemoprevention of prostate cancer: agents and study designs. J Urol. 2007;178(3 Pt 2):S9-S13.

16. Dennis LK, Snetselaar LG, Smith BJ, Stewart RE, Robbins ME Problems with the assessment of dietary fat in prostate cancer studies. Am J Epidemiol. 2004; 160(5):436-444.

17. Fleshner N, Bagnell PS, Klotz L, Venkateswaran V. Dietary fat and pros tate cancer. J Urol. 2004;171:S19-S24.

18. Wallström P, Bjartell A, Gullberg B, Olsson H, Wirfält E. A prospective study on dietary fat and incidence of prostate cancer (Malmö, Sweden). Cancer Causes Control. 2007 Dec; 18(10):1107-1121.

19. Kelavkar UP, Hutzley J, Dhir R, Kim P, Allen KG, McHugh K. Prostate tumor growth and recurrence can be modulated by the omega- 6 : omega-3 ratio in diet: athymic mouse xenograft model simulatin radical prostatectomy. Neoplasia. 2006 Feb;8(2):112-124.

20. Leitzmann MF, Stmpfer MJ, Michauk DS, Augustsson K, Colditz GC, Willett WC, Giovannucci EL. Am J Clin Nurt. 2004 Jul;80(1):204-216.

21. Kirsh VA, Hayes RB, Mayne ST, Chatterjee N, Subar AF, Dixon LB, et al. Supplemental and dietary vitamin E. beta-carotene, and vitamin C intakes and prostate cancer risk. J Natl Cancer Inst. 2006;98(4):245-254.

22. Ozmen H, Erulas FA, Karatas F, Cukurovali A, Yalcin O. Comparison of the concentration of trace metals (NI, $\mathrm{Zn}, \mathrm{Co}, \mathrm{Cu}$ and Se), Fe, vitamins $\mathrm{A}, \mathrm{C}$ and $\mathrm{E}$, an lipid peroxidation in patients with prostate cancer. Clin Chem Lab Med. 2006;44(2):175-179.

23. Miller EC, Givannucci E, Erdman JW, Bahnson R, Schwartz SJ, Clinton SK. Tomato prosucts, lycopene, and prostate cancer risk. Urol Clin North Am. 2002;29(1):83-93.

24, Itsiopoulos C, Hodge A, Kaimakamis M. Can the Mediterranean diet prevent prostate cancer? Mol Nutr Food Res. 2009;53(2):227-239.

25. Hoenjet KM, Dagnelie PC, Delaere KP, Wijchmans NE, Zambon JV, Oosterhof GO. Effect of a nutritional supplement containing vitamin $\mathrm{E}$ selenium, vitamin C and coenzyme $\mathrm{Q} 10$ on serum PSA in patients with hormonally untreated carcinoma of the prostate: a randomised placebocontrolled study. Eur Urol. 2005;47(4):433-439.

26. Gaziano JM, Glynn RJ, Christen WG, Kurth T, Belanger C, Macfadyen J, Bubes V, et al. Vitamins E and C in the Prevention of Prostate and Total Cancer in Men: The Physicians Health Study II Randomized Controlled Trial. JAMA. 2009;301(1):52-62.

27. Coulter ID, Hardy ML, Morton SC, Hilton LG, Tu W, Vaalentine D, et al. Antioxidants vitamin $\mathrm{C}$ and vitamin $\mathrm{E}$ for the prevention and treatment of cancer. J Gen Intern Med. 2006;21(7):735-744.

28. Navarro-Alarcon M, Cabrera-Vique C. Selenium in food and the human body: a review. Sci Total Environ. 2008:400(1-3):115-141.

29. Key TJ, Appleby PN, Allen NE, Travis RC, Roddam AW, Jenab M, et al. Plasma carotenoids, retinol, and tocopherols and the risk of prostate cancer in the European Prospective Investigation into Cancer and Nutrition study. Am J Clin Nutr. 2007; 86(3):672-81.

Correspondencia autora: Prof. Constanza López Fontana Laboratorio de Enfermedades Metabólicas y Cáncer. Facultad de Farmacia y Bioquímica. Universidad Juan Agustín Maza. Acceso Este Lateral Sur 2245.

(CP 5519) Guaymallén. Mendoza , Argentina.

Tel. +54-261-4257535

E-mail autora: investigacioncap@yahoo.com.ar Información artículo: Original - Cáncer de próstata Trabajo recibido: enero 2009

Trabajo aceptado: abril 2009 